\title{
Article
}

\section{Umbral Methods and Harmonic Numbers}

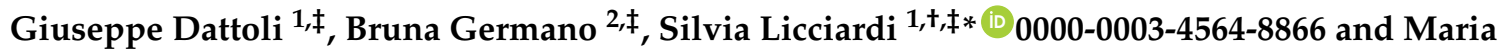 Renata Martinelli ${ }^{2, \ddagger}$}

1 ENEA - Frascati Research Center, Via Enrico Fermi 45, 00044, Frascati, Rome, Italy; giuseppe.dattoli@enea.it, silviakant@gmail.com

2 University of Rome, La Sapienza, Department of Methods and Mathematic Models for Applied Sciences, Via A. Scarpa, 14, 00161 Rome, Italy, bruna.germano@sbai.uniroma1.it, martinelli@dmmm.uniroma1.it

* Correspondence: silviakant@gmail.com; Tel.: +39-392-509-6741

$\dagger$ Current address: ENEA - Frascati Research Center, Via Enrico Fermi 45, 00044, Frascati, Rome, Italy

$\ddagger$ These authors contributed equally to this work.

\begin{abstract}
The theory of harmonic based function is discussed here within the framework of umbral operational methods. We derive a number of results based on elementary notions relying on the properties of Gaussian integrals.
\end{abstract}

Keywords: Harmonic Numbers 11K99, Operators 44A99, 47B99, Umbral methods 05A40, Special Functions 33C52, 33C65, 33C99, 33B10, 33B15, Hermite Polynomials 33C45.

\section{Introduction}

Methods employing the concepts and the formalism of umbral calculus have been exploited in [1] to guess the existence of generating functions involving Harmonic Numbers [2]. The conjectures put forward in [1] have been proven in [3]-[4], further elaborated in subsequent papers [5] and generalized to Hyper-Harmonic Numbers in [6].

In this note we use the same point of view of [1], by discussing the possibility of exploiting the formalism developed therein in a wider context.

\section{Harmonic Numbers and Generating Functions}

We remind that harmonic numbers are defined as [2]

$$
h_{n}:=\sum_{r=1}^{n} \frac{1}{r}, \quad \forall n \in \mathbb{N}
$$

It is furthermore evident that the integral representation for this family of numbers can be derived using a standard procedure, reported below.

Proposition 1. By applying the Laplace transform, we obtain

$$
h_{n}=\sum_{r=1}^{n} \int_{0}^{\infty} e^{-s r} d s
$$

thereby getting [7] the Euler's integral

$$
h_{n}=\int_{0}^{1} \frac{1-x^{n}}{1-x} d x
$$


Proof.

$$
\begin{aligned}
h_{n} & =\sum_{r=1}^{n} \int_{0}^{\infty} e^{-s r} d s=\int_{0}^{\infty}\left[\left(\sum_{r=0}^{n} e^{-s r}\right)-1\right] d s= \\
& =\int_{0}^{\infty} \frac{1-\left(e^{-s}\right)^{n+1}}{1-e^{-s}}-1 d s=\int_{-\infty}^{0} \frac{1-\left(e^{s}\right)^{n+1}}{1-e^{s}}-1 d s= \\
& =\int_{-\infty}^{0} \frac{e^{(n+1) s}-e^{s}}{e^{s}-1} d s
\end{aligned}
$$

and by applying the change of variables $e^{s} \rightarrow x$ we obtain

$$
h_{n}=\int_{0}^{1} \frac{1-x^{n}}{1-x} d x
$$

20 The definition in eq. (3) can be extended to non-integer values of $n$ and, therefore, it can be exploited as an alternative definition holding for $n$ (not-necessarily) a positive real.

Definition 1. The function

$$
\varphi_{h}(z):=\int_{0}^{1} \frac{1-x^{z}}{1-x} d x, \quad \forall z \in \mathbb{R}
$$

23 is called harmonic number umbral vacuum, or simply the vacuum.

Definition 2. The operator

$$
\hat{h}:=e^{\partial_{z}}
$$

24 is the vacuum shift operator.

Theorem 1. The umbral operator, $\hat{h}^{n}$, defines the harmonic numbers, $h_{n}$, as the action of the operator (5) on the vacuum (4):

$$
\left.\hat{h}^{n} \varphi_{h}(z)\right|_{z=0}=h_{n}
$$

or simply

$$
\begin{aligned}
& \hat{h}^{n}=h_{n}, \\
& h_{0}=0
\end{aligned}
$$

\section{Proof.}

$$
\begin{aligned}
\left.\hat{h}^{n} \varphi_{h}(z)\right|_{z=0} & =\left.e^{n \partial_{z}} \varphi_{h}(z)\right|_{z=0}=\left.\varphi_{h}(z+n)\right|_{z=0}=\left.\int_{0}^{1} \frac{1-x^{z+n}}{1-x} d x\right|_{z=0}= \\
& =\int_{0}^{1} \frac{1-x^{n}}{1-x} d x=h_{n}
\end{aligned}
$$

\section{Properties 1.}

$$
\hat{h}^{n} \hat{h}^{m}=\hat{h}^{n+m}
$$

The proof follows from eq. (5). We just note that $n, m$ are not necessarily positive integers.

Definition 3. We call Harmonic Based Exponential Function (HBEF) the series

$$
{ }_{h} e(x):=e^{\hat{h} x}=1+\sum_{n=1}^{\infty} \frac{h_{n}}{n !} x^{n}
$$


27

28

29 The relevant derivatives can accordingly be expressed as (see the concluding part of the paper for

so further comments)

$$
\begin{aligned}
& \left(\frac{d}{d x}\right)^{m}{ }_{h} e(x):={ }_{h} e(x, m)=\hat{h}^{m} e^{\hat{h} x}=h_{m}+\sum_{n=1}^{\infty} \frac{h_{n+m}}{n !} x^{n}, \\
& \left(\frac{d}{d x}\right)^{m}{ }_{h} e(x, k)={ }_{h} e(x, k+m)
\end{aligned}
$$

31

and, according to eq. (9) we also find that

$$
\int_{0}^{\infty} h^{e}(-\alpha x) e^{-x} d x=\int_{0}^{\infty} e^{-(\alpha \hat{h}+1) x} d x=\frac{1}{\alpha \hat{h}+1}
$$

32 Corollary 1. By expanding the umbral function on the r.h.s. of eq. (11), we obtain

$$
\frac{1}{\alpha \hat{h}+1}=1+\sum_{n=1}^{\infty}(-1)^{n} \alpha^{n} h_{n}
$$

Proof. Using the Taylor expansion and the eq. (7), we have

$$
\frac{1}{\alpha \hat{h}+1}=\sum_{n=0}^{\infty}(-\alpha \hat{h})^{n}=1+\sum_{n=1}^{\infty}(-1)^{n} \alpha^{n} \hat{h}^{n}=1+\sum_{n=1}^{\infty}(-1)^{n} \alpha^{n} h_{n}
$$

\section{Example 1.}

$$
\int_{-\infty}^{\infty} h e(-\alpha x) e^{-x^{2}} d x=\int_{-\infty}^{\infty} e^{-\left(\alpha \hat{h} x+x^{2}\right)} d x=\sqrt{\pi} e^{\alpha^{2} \hat{h}^{2}}
$$

37 The last term in eq. (13) has been obtained by treating $\hat{h}$ as an ordinary algebraic quantity and then by 38 applying the standard rules of the Gaussian integration.

We notice that, using eq. (9), we obtain

$$
h^{2} e\left(\frac{\alpha^{2}}{4}\right):=e^{\frac{h^{2} \alpha^{2}}{4}}=1+\sum_{r=1}^{\infty} \frac{h_{2 r}}{r !}\left(\frac{\alpha}{2}\right)^{2 r}
$$

39 Let us now consider the following slightly more elaborated example, involving the integration of 40 two "Gaussians", namely the ordinary case and its HBEF analogous.

\section{Example 2.}

$$
\int_{-\infty}^{\infty} h e\left(-\alpha x^{2}\right) e^{-x^{2}} d x=\int_{-\infty}^{\infty} e^{-(\hat{h} \alpha+1) x^{2}} d x=\sqrt{\frac{\pi}{1+\alpha \hat{h}}}
$$

This last result, obtained after applying elementary rules, can be worded as it follows: the integral in eq. (15) depends on the operator function on its r.h.s., for which we should provide a computational meaning. The use of the Newton binomial yields 


$$
\begin{aligned}
& \sqrt{\frac{\pi}{1+\alpha \hat{h}}}=\sqrt{\pi} \sum_{r=0}^{\infty}\left(\begin{array}{c}
-\frac{1}{2} \\
r
\end{array}\right)(\alpha \hat{h})^{r}=\sqrt{\pi}\left(1+\sqrt{\pi} \sum_{r=1}^{\infty} \frac{\alpha^{r} h_{r}}{\Gamma\left(\frac{1}{2}-r\right) r !}\right), \\
& |\alpha|<1
\end{aligned}
$$

45 The correctness of this conclusion has been confirmed by the numerical check.

47

defines a $H B E F$ through the following Gauss transform

$$
\int_{-\infty}^{+\infty} \sqrt{h} e(\alpha x) e^{-x^{2}} d x=\int_{-\infty}^{+\infty} e^{\hat{h}^{\frac{1}{2}} \alpha x-x^{2}} d x=\sqrt{\pi} e^{\hat{h}\left(\frac{\alpha}{2}\right)^{2}}=\sqrt{\pi}_{h} e\left(\left(\frac{\alpha}{2}\right)^{2}\right)
$$

${ }_{55}$ On the other side, the function (17) can be expressed in terms of the $\mathrm{HBEF}_{{ }_{h}} e(x)$, using appropriate 56 integral transform methods [8].

Definition 4. Let

$$
g_{\frac{1}{2}}(\eta)=\frac{1}{2 \sqrt{\pi \eta^{3}}} e^{-\frac{1}{4 \eta}}
$$

57 the Levy distribution of order $\frac{1}{2}$ then [8]

$$
e^{-p^{\frac{1}{2}} x}=\int_{0}^{\infty} e^{-p \eta x^{2}} g_{\frac{1}{2}}(\eta) d \eta
$$

58 is the associated Levy integral transform.

59 The use of eq. (17) allows to write the identity

\section{Corollary 2.}

$$
\begin{aligned}
& { }_{\sqrt{h}} e(-x)=\int_{0}^{\infty} h e\left(-\eta x^{2}\right) g_{\frac{1}{2}}(\eta) d \eta, \\
& g_{\frac{1}{2}}(\eta)=\frac{1}{2 \sqrt{\pi \eta^{3}}} e^{-\frac{1}{4 \eta}}
\end{aligned}
$$

Proof.

$$
\sqrt{h}^{h} e(-x)=e^{-\hat{h}^{\frac{1}{2}} x}=\int_{0}^{\infty} e^{-\hat{h} \eta x^{2}} g_{\frac{1}{2}}(\eta) d \eta=\int_{0}^{\infty} h e\left(-\eta x^{2}\right) g_{\frac{1}{2}}(\eta) d \eta
$$

60 The possibility of defining $\sqrt[k]{h}^{e}(x)$ will be discussed elsewhere.

${ }_{61}$ Theorem 2. The function ${ }_{h} e(x)$ satisfies the first order non homogeneous differential equation 


$$
\left\{\begin{array}{l}
{ }_{h} e^{\prime}(x)=\frac{d}{d x} h_{h} e(x)={ }_{h} e(x)+\frac{e^{x}-x-1}{x}, \quad \forall x \in \mathbb{R} \\
{ }_{h} e(0)=1
\end{array}\right.
$$

Proof. Eq. (10) for $m=1$ yields

$$
{ }_{h} e^{\prime}(x)={ }_{h} e(x, 1)=1+\sum_{n=1}^{\infty} \frac{h_{n+1}}{n !} x^{n}
$$

Being $h_{n+1}=h_{n}+\frac{1}{n+1}$ we find

$$
1+\sum_{n=1}^{\infty} \frac{h_{n+1}}{n !} x^{n}={ }_{h} e(x)+\frac{1}{x}\left(e^{x}-x-1\right)
$$

62 hence eq. (22) follows.

Corollary 3. The solution of eq. (22) yields for the HBEF the explicit expression in terms of ordinary special functions.

$$
\begin{aligned}
& h^{e}(x)=1+e^{x}\left(\ln (x)+E_{1}(x)+\gamma\right), \\
& E_{1}(x)=\int_{x}^{\infty} \frac{e^{-t}}{t} d t \\
& \left(\ln (x)+E_{1}(x)+\gamma\right)=-\sum_{n=1}^{\infty} \frac{(-x)^{n}}{n n !} \\
& \gamma \equiv \text { Euler - Mascheroni-constant }
\end{aligned}
$$

${ }_{63}$ The previous expression is the generating function of harmonic numbers originally derived by Gosper

64 (see [2]).

65

66 By iterating the previous procedure we find the following general recurrence

\section{Corollary 4.}

$$
{ }_{h} e(x, m)={ }_{h} e(x)+\sum_{r=0}^{m-1}\left(\frac{d}{d x}\right)^{r} \frac{e^{x}-1-x}{x}
$$

Definition 5. The binomial expansion

$$
h_{n}(x)=(x+\hat{h})^{n}=x^{n}+\sum_{s=1}^{n}\left(\begin{array}{l}
n \\
s
\end{array}\right) x^{n-s} h_{s}
$$

67 specifies the Harmonic Polynomials.

68 They are easily shown to be linked to the HBEF by means of the generating function:

\section{Corollary 5.}

$$
\sum_{n=0}^{\infty} \frac{t^{n}}{n !} h_{n}(x)=e^{x t}{ }_{h} e(t)
$$

\section{Proof.}

$$
\sum_{n=0}^{\infty} \frac{t^{n}}{n !} h_{n}(x)=\sum_{n=0}^{\infty} \frac{t^{n}}{n !}(x+\hat{h})^{n}=e^{t(x+\hat{h})}=e^{x t}{ }_{h} e(t)
$$


69 They belong to the family of Appél polynomials and satisfy the recurrences:

\section{Properties 2.}

$$
\begin{aligned}
& \text { i) } \frac{d}{d x} h_{n}(x)=n h_{n-1}(x) \\
& \text { ii) } h_{n+1}(x)=(x+1) h_{n}(x)+f_{n}(x) \text {, } \\
& f_{n}(x):=\sum_{s=1}^{n} \frac{n !}{(n-s) !} \frac{x^{n-s}}{(s+1) !}=\int_{0}^{1}(x+y)^{n} d y-x^{n}
\end{aligned}
$$

Proof. The derivation of eq. (29) is trivial; regarding eq. (30) we have:

$$
\begin{aligned}
h_{n+1}(x) & =(x+\hat{h})(x+\hat{h})^{n}=(x+\hat{h})\left(x^{n}+\sum_{s=1}^{n}\left(\begin{array}{l}
n \\
s
\end{array}\right) x^{n-s} \hat{h}^{s}\right)= \\
& =x h_{n}+1 \cdot x^{n}+\sum_{s=1}^{n}\left(\begin{array}{l}
n \\
s
\end{array}\right) x^{n-s} \hat{h}^{s+1}= \\
& =x h_{n}(x)+\left(x^{n}+\sum_{s=1}^{n}\left(\begin{array}{l}
n \\
s
\end{array}\right) x^{n-s} \hat{h}^{s}\right)+\sum_{s=1}^{n} \frac{n ! x^{n-s}}{(n-s) !(s+1) !}= \\
& =(x+1) h_{n}(x)+\sum_{s=1}^{n} \frac{n ! x^{n-s}}{(n-s) !(s+1) !}
\end{aligned}
$$

and

$$
\begin{aligned}
& \sum_{s=1}^{n} \frac{n !}{(n-s) !} \frac{x^{n-s}}{(s+1) !}=\left.\sum_{s=1}^{n} \frac{n !}{s !(n-s) !} \frac{x^{n-s}}{s+1} y^{s+1}\right|_{y=1}= \\
& =\sum_{s=1}^{n}\left(\begin{array}{l}
n \\
s
\end{array}\right) x^{n-s} \int_{0}^{1} y^{s} d y=\int_{0}^{1} \sum_{s=0}^{n}\left(\begin{array}{l}
n \\
s
\end{array}\right) x^{n-s} y^{s}-x^{n} d y= \\
& =\int_{0}^{1}(x+y)^{n} d y-x^{n}
\end{aligned}
$$

Corollary 6. The identity

$$
h_{n}(-1)=(-1)^{n}\left(1-\frac{1}{n}\right)
$$

follows from the eq. (30) after setting $x=-1$.

The identity

$$
h_{n}=1+\sum_{s=1}^{n}\left(\begin{array}{l}
n \\
s
\end{array}\right) h_{s}(-1)
$$

71 is a consequence of the fact that $\hat{h}^{n}=((\hat{h}-1)+1)^{n}$.

72 The harmonic Hermite polynomials (touched on in ref. [1]-[3]-[9]) can also be written as

\section{Definition 6.}

$$
\begin{aligned}
& \sum_{n=0}^{\infty} \frac{t^{n}}{n !}{ }_{h} H_{n}(x)=e^{x t}{ }_{h} e\left(t^{2}\right) \\
& { }_{h} H_{n}(x):=H_{n}(x, \hat{h})=e^{\hat{h} \partial_{x}^{2}} x^{n}=x^{n}+n ! \sum_{r=1}^{\left\lfloor\frac{n}{2}\right\rfloor} \frac{x^{n-2 r} \hat{h}^{r}}{(n-2 r) ! r !}
\end{aligned}
$$


Properties 3. The recurrences identity of the umbral Hermite polynomials

$$
\begin{aligned}
& \text { i) } \frac{d}{d x}{ }_{h} H_{n}(x)=n_{h} H_{n-1}(x) \\
& \text { ii) }{ }_{h} H_{n+1}(x)=\left(x+2 \hat{h} \frac{d}{d x}\right){ }_{h} H_{n}(x)=\left(x+2 \frac{d}{d x}\right){ }_{h} H_{n}(x)+2 \alpha_{n}^{\prime}(x) \\
& \alpha_{n}(x)=n ! \sum_{s=1}^{\left\lfloor\frac{n}{2}\right\rfloor} \frac{x^{n-2 s}}{(s+1) !(n-2 s) !} \\
& \alpha_{n}^{\prime}(x)=\frac{d}{d x} \alpha_{n}(x)
\end{aligned}
$$

73

\section{Final Comments}

Before closing the paper, we want to stress the possibility of extending the present procedure to the truncated exponential numbers, namely

$$
e_{n}:=\sum_{r=0}^{n} \frac{1}{r !}
$$

76 The relevant integral representation writes [11]

$$
e_{\alpha}:=\frac{1}{\Gamma(\alpha+1)} \int_{0}^{\infty} e^{-s}(1+s)^{\alpha} d s
$$

77 which holds for non-integer real values of $\alpha$ too. For example we find

\section{Example 3.}

$$
e_{-\frac{1}{2}}=\frac{e}{\sqrt{\pi}} \Gamma\left(\frac{1}{2}, 1\right)
$$

78 with $\Gamma\left(1, \frac{1}{2}\right)$ being the truncated Gamma function.

According to the previous discussion and to eq. (38), setting $\hat{e}^{\alpha} \leftrightarrow e_{\alpha}$, we also find that

$$
\begin{aligned}
& \int_{-\infty}^{+\infty} e^{-\hat{e} x^{2}} d x=\sqrt{\pi} e_{-\frac{1}{2}} \\
& e^{-\hat{e} x^{2}}=\sum_{r=0}^{\infty}(-1)^{r} \frac{e_{r}}{r !} x^{2 r}
\end{aligned}
$$

79 This last identity is a further proof that the implications offered by the topics treated in this paper are fairly interesting and deserve further and more detailed investigation, which will be more accurately treated elsewhere.

82 
2. J. Sondow, E.W. Weisstein, "Harmonic Number", Math World A Wolfram Web Resource, 87 http://mathworld.wolfram.com/ HarmonicNumber.html.

88 3. M.W. Coffee, "Expressions for Harmonic Number Generating Functions", Contemporary Mathematics, 517, Gems

89 In Experimental Mathematics, T. Amdeberhan, E.T. Simos, V.H. Moll eds., AMS Special Session Experimental 9o Mathematics, (2009).

91 4. D. Cvijović, "The Dattoli-Srivastava Conjectures Concerning Generating Functions Involving the Harmonic Numbers", 92 Appl. Math. Comput., 215(9), pp. 4040-4043, (2010).

93 5. I. Mezo, "Exponential Generating Function of Hyper-Harmonic Numbers Indexed by Arithmetic Progressions", Cent.

$94 \quad$ Eur. J. Math., 11(3), pp. 931-939, (2013).

95 6. J.H. Conway, R.K. Guy, "The Book of Numbers", Copernicus, New York, (1996).

96 7. J.A. Rochowicz Jr, "Harmonic Numbers: Insights, Approximations and Applications", Spreadsheets in Education 97 (eJSiE): Vol. 8: Iss. 2, Article 4, (2015).

98 8. G. Doetsch, "Handbuch der Laplace Transformation", Birkhnauser, Basel, (1950-1956).

99 9. K. Zhukovsky, G. Dattoli, "Umbral Methods, Combinatorial Identities And Harmonic Numbers", Applied 100 Mathematics, 1, 46 (2011).

101 10. G. Dattoli, "Generalized Polynomials, Operational Identities and their Applications", J. Comput Appl Math.,Elsevier 102 Volume 118, Issues 12, 1 June 2000, Pages 111-123. doi: 10.1016/S0377-0427(00)00283-1.

103 11. G. Dattoli, P.E. Ricci, L. Marinelli, "Generalized Truncated Exponential Polynomials and Applications", Rendiconti 104 dell'Istituto di Matematica dell’Universitá di Trieste, An International Journal of Mathematics, 34, pp. 9-18, 105 (2002). 